\title{
Discriminación y violencia sexualizada en las Instituciones de Educación Superior en Alemania. Estrategias de intervención y desafíos persistentes
}

\section{Sexual discrimination and violence in the German institutions of higher education. Intervention strategies and ongoing challenges.}

\author{
Nadja Damm ${ }^{1^{*}}$
}

Fecha de recepción: 10 de enero de 2019

Fecha de aceptación: 9 de agosto de 2019

\begin{abstract}
Resumen - La discriminación y violencia sexualizada (DVS) es un problema que ocurre en las Instituciones de Educación Superior (IES) en todo el mundo. En Alemania, según un estudio que se hizo a nivel europeo en 2012, $54.7 \%$ de las estudiantes enfrentaron acoso sexual en el ámbito universitario y 3.3\% fueron víctimas de violencia sexual. Como respuesta a esta problemática, las universidades alemanas han ido estableciendo mecanismos de prevención, intervención y sanción, y las estudiantes y activistas feministas han desarrollado sus propias estrategias para sensibilizar, intervenir y apoyarse mutuamente.

En el artículo se presentarán los datos centrales sobre DVS en la educación superior en Alemania y las diversas tácticas y medidas establecidas en contra de esta conducta (con un enfoque en los protocolos y sus bases jurídicas). Al final se resumen las acciones emprendidas y las problemáticas persistentes con miras a llegar a un sistema de educación superior exento de discriminación y violencia sexualizada que permita a todas sus integrantes desarrollar libremente sus conocimientos, sus competencias y su personalidad.
\end{abstract}

\section{Palabras clave:}

Discriminación y violencia sexualizada, Alemania.
Abstract - Sexual Discrimination and Violence (SDV) is a relevant problem in college-level institutions all around the world. According to a European study carried out in 2012 , in Germany $54.7 \%$ of all female university students faced sexual harassment, and $3.3 \%$ were victims of sexual violence. Responding to this problem, German universities have introduced mechanisms of prevention, intervention and sanction, meanwhile feminist activists have developed their own strategies to raise awareness about the problem, to intervene, and to support each other.

This paper presents the main data about SDV in college-level institutions in Germany and the diverse strategies and measures developed to confront SDV (with a focus on guidelines and their legal basis). It sums up the steps already taken and the ongoing challenges on the way to achieve a higher education system free from discrimination and sexual violence, that allows all of its members to freely develop their knowledge, competencies and personality.

\section{Keywords:}

Discrimination, sexual violence, Germany. 


\section{Introducción}

ace 120 años, en Alemania pudieron ingresar las primeras mujeres a las universidades a estudiar una carrera académica. En 2018, celebramos 100 años del derecho al voto de las mujeres y hace casi 50 años, en 1970, se abrieron las primeras casas de refugio para aquellas que sufrían violencia doméstica. Hace aproximadamente 20 años, en 1997, se cambió la ley penal y desde entonces la violación en el marco del matrimonio se califica como delito penal. En 2016 se logró cambiar esta ley de nuevo, con el resultado de que ahora un no significa "no"; es decir, que según el artículo 177 de la ley penal ya basta con que una mujer diga "no" a su agresor para que una violacion u otro tipo de acoso sea reconocido como tal. ${ }^{2}$

Así que ha habido algunos cambios legislativos, políticos y culturales importantes para acercarnos más a la igualdad de género en la vida política, académica y privada. Sin embargo, para lograrlo todavía nos quedan algunas cimas que sobrepasar y estructuras culturales que cambiar.

Por si no lo habíamos percibido antes, en los últimos dos años los movimientos \#metoo y \#niunamenos nos mostraron que la discriminación y la violencia de género suceden en todo el mundo y en todos los ámbitos de la sociedad: en la esfera cultural (en la produción de películas, telenovelas y los comités del Premio Nobel de Literatura, entre otros); en la vida política, como en el parlamento europeo; en la Iglesia católica, la luterana y en otras comunidades religiosas; en las familias; en los centros educativos, desde los jardines de niños hasta la educación superior; en las instituciones de pedagogía social; en las asociaciones deportivas y en la vida laboral en general. En Alemania, en 2013 el hashtag "\#aufschrei" (\#grito) mostró lo "normal" que sigue siendo el sexismo en la vida cotidiana. En 2016 el hashtag "\#ausnahmslos" (\#sinexcepción) exigió la intervención ante cada tipo de hostigamiento y acoso sexual, al tiempo que criticó la instrumentalización de este tema por motivos racistas. ${ }^{3}$

El hostigamiento y acoso sexual (HAS) así como la discriminación y violencia sexualizada (DVS) contra las mujeres y contra personas de otros géneros y de todas las edades -como una forma específica de violencia de género- se presenta no solamente en las IES, sino en todos los ámbitos privados y públicos, pues en todos los contextos nos encontramos con las estructuras discriminatorias del sexismo y heterosexismo (que muchas veces son intersectadas con otras formas discriminatorias, como el racismo, clasismo, adultismo o la discriminación de personas de diversidad sexual o discapacitadas). Y ocurre en los entornos donde haya relaciones jerárquicas de poder que pueden ser usadas como medio para lograr sus fines.

Con este artículo ${ }^{4}$ quiero dar un panorama de la investigación sobre la DVS en la educación superior de Alemania, presentando datos centrales sobre el fenómeno y algunas medidas ejemplares de prevención, intervención y sanción. Partiendo de esta introducción, se hará una breve aclaración de términos, luego se

${ }^{2}$ Antes se requería que la víctima pusiera resistencia durante la agresión y que hubiera una amenaza o violencia por parte del acusado (Sanyal, 2016, p. 165f.).

${ }^{3}$ En la noche de año nuevo de 2015 a 2016, en Colonia y otras ciudades alemanas en las fiestas públicas sucedieron varios actos de hostigamiento sexual, muchos de los cuales fueron efectuados por hombres que parecían ser refugiados de países árabes y de África del Norte. En los medios de comunicación esto provocó un clima de acusación general hacia todos los varones migrantes y una instrumentalización racista del tema por parte de grupos y partidos de derecha. No se habló de la DVS que sucede en la vida cotidiana y que proviene de agresores alemanes, sino en el discurso se dio la imagen de que la DVS era "un problema importado"con los refugiados y hombres migrantes. Es por eso que feministas de diferentes corrientes y orígenes crearon el hashtag "\#sinexcepción", para enfatizar que ellas luchan en contra de la DVS sin excepción; es decir, también en contra de las agresiones sexualizadas causadas por individuos blancos y alemanes (Sanyal, 2016, p. 108 y ss).

${ }^{4}$ Este texto se basa en una ponencia que dicté en la "Primera Reunión Nacional sobre mecanismos contra el hostigamiento y acoso sexual en Instituciones de Educación Superior", que se llevó a cabo en la UABJO en noviembre de 2018. Quiero agradecer a las organizadoras de esta conferencia por convocar al encuentro, por darme la oportunidad de participar, y a todas las participantes, compañeras de trabajo y de activismo feminista por intercambiar conocimientos, sentimientos, experiencias, ideas y estrategias. Mi motivación actual de dedicarme al tema y de tomar parte en la conferencia tiene que ver con un caso en un proyecto de cooperación entre la ASH Berlín y la UABJO. En febrero de 2017 hubo una denuncia de violación en contra de un educador de la UABJO que también estaba trabajando en los proyectos de cooperación con la ASH Berlín; me enteré por dos estudiantes alemanas que estuvieron en Oaxaca en el marco de la cooperación cuando se publicó la denuncia y también a través del blog feminismos en debate, del grupo MUOVAS. 
presentarán los datos centrales sobre la DVS en las IES alemanas, para de ahí dar una idea de las bases jurídicas de los mecanismos y las estrategias ejemplares. Finalmente, a modo de conclusión, se resumirán los pasos ya tomados y las problemáticas persistentes para lograr un sistema de educación superior exento de discriminación y violencia de género que permita a todas sus integrantes desarrollar libremente sus concimientos, competencias y personalidad.

\section{Términos: hostigamiento, acoso y violencia sexualizada}

En Alemania, los términos "hostigamiento y acoso sexual (HAS)" y "discriminación y violencia sexual" (DVS) se usan para nombrar las formas específicas del concepto general de discriminación y violencia de género que se muestran de manera sexualizada. En los últimos años se acostumbra más hablar de violencia "sexualizada" en lugar de "sexual" para cambiar la perspectiva y hacer hincapié en que no se trata de una forma de sexualidad, sino más bien de un acto violento y un abuso de poder que ha sido sexualizado.

Refiriéndose al artículo 3, párrafo 4 de la Ley de Igualdad de Trato (AGG, 2006, capítulo 4), la coordinadora de las delegadas de la mujer de todas las IES en Alemania (Bukof,, en adelante la "coordinadora") define al HAS y a la DVS como un comportamiento sexualizado indeseado por la persona afectada, que tiene como fin o efecto lastimar su dignidad. Estas conductas o acciones pueden ser verbales, no verbales y corporales, es decir, agresiones físicas. La definición según este artículo implica que no es necesario que el agresor tenga conciencia de su ataque o que lo haga con intención, basta con que la víctima perciba su acto como humillación o lesivo (Kocher y Porsche, 2015, p. 10). ${ }^{6}$
Hasta ahora todas las investigaciones en Alemania indican que las personas afectadas por la DVS son mujeres* y también lesbianas, gays, bisexuales, transexuales, transgender, intersexuales y queers (LGBTIQ*).7 Es decir, tanto mujeres* como la gente discriminada por su identidad de sexo o género o por su orientación sexual, además de los varones que son percibidos como diferentes al estereotipo masculino, sufren DVS (Bukof, 2018a, p. 2).

\section{Datos: DVS en las IES de Alemania, un doble tabú}

La discriminación y violencia sexualizada (no solamente) en las IES puede tener consecuencias graves: las personas afectadas posiblemente ya no se desplazan libremente en ciertos espacios, ya no van a clases o a su trabajo, padecen problemas psicológicos y hasta síndromes de estrés postraumático. Muy probablemente su rendimiento universitario o laboral disminuye, se retrasan en el proceso de sus estudios 0 hasta abandonan la universidad sin terminar la carrera o sin haber encontrado otro puesto de trabajo, tal vez con deudas y con el sentimiento de haber fallado (Kocher y Porsche, 2015, p. 8; Feltes, List, Schneider \& Höfker, 2012, p. 5).

Hablando de DVS en general, la última Dunkelfeldstudie (estudio representativo sobre la cifra negra) realizada por el Ministerio Federal de la Mujer en 2004 , indica que $13 \%$ de las mujeres* (mayores de 16 años) ha padecido violencia sexualizada y $58 \%$ discriminación sexualizada (BMFSFJ, 2008, p. 7). El porcentaje de las que sobreviven a una violación y no la reportan a la policía o fiscalía está entre 82 y 95\% (Bff, s.f; Stadler Bieneck \& Pfeiffer, 2012; Wiezorek, 2014, p. $121)$. Las razones son diversas, entre las que destacan

\footnotetext{
${ }^{5}$ Bukof es la abreviación de "Bundekonferenz der Frauenbeauftragten", que significa "coordinadora de las delegadas de la mujer de todas las IES". ${ }^{6}$ Esta definición también se está contemplando en la mayoría de los protocolos.

${ }^{7}$ Usando asterisco en mujer* y en LGBTIQ* se indica que aunque una persona parezca ser de determinado sexo, puede ser que ella misma se considere de otro; también para señalar que no solamente existen los sexos masculino y femenino, sino toda una variedad. En este texto voy a utilizar solamente la forma femenina, aunque debería de emplear también la masculina y una forma para todas las personas que se consideran de otro género, aparte de ser hombres o mujeres.
} 
el miedo de ser estigmatizadas y/o revictimizadas en el proceso jurídico, la falta de confianza de ser protegidas por las instituciones estatales y el conocimiento de la alta impunidad en cuanto a las violaciones (Eberlei, Neuhoff \& Eberlei, 2018, p. 87; Oerter, Lorenz \& Kleine, 2012; Seifarth \& Ludwig, 2016). La cantidad de acusaciones que resultan ser falsas en Europa está entre $1 \%$ y $9 \%$, mientras que en Alemania llega a $3 \%$ (Wiezorek, 2014, p. 121).

Actualmente, tomando en cuenta la cifra negra, solamente en $0.7-1.4 \%$ de los casos los agresores son condenados, por lo que se puede hablar de una impunidad severa en Alemania (Wiezorek, 2014, p. 121). Se espera que con los cambios en la ley penal (art. 177) de 2016 este porcentaje aumente.

Mechtild Korueber, delegada de la mujer* en la Universidad Libre de Berlín (FU Berlín ${ }^{8}$ ), habla de un doble tabú en el tema: primero, porque la DVS (también hacia los menores de edad) sigue siendo un secreto a voces en toda la sociedad alemana; y segundo, porque en las universidades todavía prevalece la actitud de que "en nuestra institución no existe este problema". También otras delegadas ${ }^{9}$ reportan que cuando empezaron a implementar protocolos en sus instituciones fueron confrontadas con la actitud de los rectorados (y otros actores) de que este problema no existía en la universidad (Kocher \& Porsche, 2015, p. $39)$, pero el hecho de que no se reporte DVS no quiere decir que no suceda.

La primera investigación que nos aportó datos cuantitativos y cualitativos sobre el asunto (Bukof, 2018a) fue un estudio que se hizo a nivel europeo en 2012, según el cual, en Alemania $54.7 \%$ de las estudiantes viven discriminación sexualizada en el ámbito universitario, $22.8 \%$ enfrenta situaciones de persecución (stalking) y 3.3\% es víctima de violencia sexualizada (Feltes et al., 2012, p. 19). ${ }^{10}$ Asimismo, se reveló que las estudiantes son más afectadas que las mujeres de otras edades y estatus (Feltes et al., 2012 , p. 19). Como ya se mencionó, no solamente las mujeres* son agredidas, también quienes "no caben" dentro del sistema de heteronormatividad (Butler, 2004) y los varones que no siguen los esterotipos y normas de cómo debe ser "un hombre" son posibles víctimas de este tipo de violencia y discriminación (FRA, 2013, p.19).

En cuanto a los agresores, la mayoría son hombres: $97.5 \%$ de los casos de hostigamiento y $96.6 \%$ de los acosos son efectuados por ellos (Feltes et al., 2012, p. 26).

La coordinadora está convencida de que la cifra negra es alta. Hasta ahora muy pocos casos son reportados a las oficinas de quejas universitarias, las personas afectadas más bien buscan ayuda con organizaciones externas, como teléfonos de auxilio u ONGs que dan asesoría jurídica y psicológica (Bukof, 2018a, p. 2). El estudio de Kocher \& Porsche (2015, p. 39) reveló que una de las razones más importantes por las cuales casi no se había reportado casos en las instituciones mismas, es porque las víctimas no sabían dónde reportar. Una investigacion de la Agencia Europea de Derechos Fundamentales de 2013 (FRA, 2013, p. 14) llegó a la misma conclusión y una encuesta que se hizo en la universidad de la ciudad de Kiel indicó que $84.8 \%$ de las estudiantes no conocía las oficinas y personas responsables de recibir las denuncias y de brindar asesoría (Klein \& Rebitzer, 2012).

Como respuesta a esta problemática, tanto las personas afectadas de manera autónoma como

\footnotetext{
${ }^{8}$ La FU Berlín es una de las tres principales universidades de la capital alemana.

${ }^{9}$ Cabe mencionar que -de acuerdo con ciertas leyes estatales sobre las Instituciones de Educación Superior- las delegadas de la mujer en algunas IES son las actoras centrales para garantizar que se prohíba el HAS. Las leyes de otros estados describen su funcion más bien como de aconsejar y apoyar al rectorado y a otras instancias universitarias en cuestiones de equidad de género (Kocher\& Porsche, 2015, p. 25). En ambos casos, es responsabilidad de la universidad imponer la prohibición de la discriminación sexualizada y son las delegadas a quienes las personas afectadas se pueden dirigir para recibr apoyo y poner una demanda (Kocher \& Porsche, 2015, p. 26).

${ }^{10}$ Esta diferencia entre 3.3\% en el ámbito académico y $13 \%$ en la sociedad en general (véase arriba) probablemente se debe a dos razones: primero, a la cifra negra en el ámbito universitario que en este estudio no se toma en cuenta y segundo, el hecho de que 70 a $80 \%$ de las violaciones suceden en los ámbitos privados y en el marco de relaciones con personas conocidas (Wiezorek, 2014, p. 116).
} 
las universidades alemanas han ido desarrollando estrategias y estableciendo mecanismos de prevención, intervención y sanción de la DVS, basados en diferentes leyes que a continuación se presentarán brevemente.

\section{Bases jurídicas: normas federales, estatales y universitarias}

La Constitución alemana (Grundgesetz, GG, 1949) exige proteger a los individuos de la violencia y discriminación sexualizada de cualquier tipo: en el artículo 2, párrafo 1 y artículo 1 párrafo 1 se establece el derecho de personalidad y en el artículo 3 se garantiza la imperatividad de la igualdad de trato.

En los protocolos y otros mecanismos contra la DVS, además, se hace referencia al Código Penal y Civil alemán, ${ }^{11}$ a diferentes normas y protocolos de la Unión Europea ${ }^{12}$ y a las leyes de los 16 estados de la República Alemana sobre el sistema de educación superior y la equidad de género. Otra base jurídica muy importante es la Ley Federal General de Igualdad de Trato (Allgemeines Gleichbehandlungsgesetz, AGG) que se instauró en 2006 para proteger a todas las personas que sufren discriminación por su género, orientación sexual, etnicidad, religión o cosmovisión, edad o condición física y/o cognitiva (por ser discapacitados), que abarca el ámbito laboral y civil. Esta ley, en su artículo 3, párrafo 4 (AGG, 2006) indica que también la DVS es considerada explícitamente como violación del derecho individual a ser tratados sin discriminación. ${ }^{13}$
En lo que respecta a las IES existe, sin embargo, la problemática de que la AGG (2006) solamente protege a sus empleadas y empleados. Las estudiantes únicamente son defendidas en cuanto al acceso a la universidad, ya estando dentro de la misma no caben bajo el amparo de esta ley y por eso ahí hay un vacío. Las normativas más importantes y más seguras para brindarles protección no sólo a los trabajadores, sino también a las y los estudiantes, son los protocolos que se basan en el derecho de las IES a su propia legislación, en adición a las leyes estatales ${ }^{14} \mathrm{y}$ federales.

\section{Estrategias: campañasy mecanismos de prevención, intervención y sanción}

Dependiendo (no solamente) del estatus dentro o fuera de la IES, se están diseñando diferentes estrategias y tomando medidas para enfrentar y superar la DVS. Al igual que en México, también en Alemania (no únicamente) las estudiantes y activistas feministas -que no siempre son integrantes de las IESoptan, por ejemplo, por formar grupos de mujeres* 0 abrir páginas en internet para interactuar y apoyarse mutuamente, así como para emprender acciones de alcance público, mientras que (no solamente) las empleadas de administración y del cuerpo académico trabajan por cambiar las estructuras y cultura dentro de la institución, organizando campañas de sensibilización o instalando protocolos, entre otros mecanismos.

11 El Código Penal (StGB) abarca formas muy severas de violencia, como la coacción y la violación (párr. 174 ff. Strafgesetzbuch/StGB), abuso sexual de súbditos (párr. 177 Abs. 2 StGB) y persecución/ stalking (párr. 238 StGB). El Código Civil prevé la posibilidad de pedir una indemnización, argumentando que el HAS lleva consigo una violación a la dignidad de la persona y significa una violación al derecho de integridad de la personalidad (artículo 1 , GG). 12 Por ejemplo, los artículos 2 y 3 del contrato de la Unión Europea estipulan la igualdad de trato de género, y los artículos 21 y 23 de la Declaración Europea de los Derechos Fundamentales prohíben todo tipo de discriminación por cuestiones de sexo y orientación sexual (Kocher \& Porsche, 2015, p. 13). 13 Según esta Ley de Igualdad de Trato (AGG), la discriminación de género también abarca la discriminación por transición de sexo y por intersexualidad. Luego, basándose en diversos protocolos de la UE, se puede argumentar que la discriminación de sexo también protege a personas queers, transgender y a las no-binarias, es decir, a quienes no se identifican ni con el sexo masculino ni con el femenino (Kocher \& Porsche, 2015, p. 9).

${ }^{14}$ En Alemania tenemos un sistema federal con 16 estados y éstos son autónomos en su política y legislación en el área de educación. Por lo tanto, cada estado tiene su ley sobre las IES. Hasta ahora, la ley en 12 de ellos incluye un artículo que estipula la obligación de las instituciones de garantizar la equidad de género y en algunos (también en la Ley Berlinesa, BerlHG art. 5a) se menciona explícitamente la protección de los miembros de la universidad ante el HAS. Luego, cada estado tiene una Ley Estatal de Equiparidad de Género, que también funciona como base jurídica de los mecanismos en contra de estas conductas. Con estas leyes, por lo menos en el caso de Berlín (Landesgleichstellungsgesetz, art. 12), tenemos el mismo problema como con la AGG (la Ley de Igualdad de Trato), que sólo comprende el ámbito laboral y por lo tanto no brinda protección a las estudiantes. 


\section{Los protocolos}

En Alemania hay 415 IES. ${ }^{15}$ La Conferencia de todos los rectorados (Hochschulrektorenkonferenz/ Conferencia), en su reunión de abril de 2018, publicó la recomendación "En contra de la discriminación y el acoso sexualizado en las escuelas superiores" (Conferencia, 2018). En ella pone énfasis en la responsabilidad de las instituciones de proteger a todas sus integrantes e implementar protocolos con tal fin; no obstante, hasta ahora no hay datos del número de las que ya cuentan con alguno. En el sitio web de la coordinadora se encuentran 30 protocolos de diferentes universidades y escuelas superiores (Bukof, 2018c).

En dichos protocolos se proponen medidas de sensibilización y prevención, y sobre todo, se aclaran y definen los procedimientos de intervención, además de las medidas de sanción. Los procesos a seguir ayudan a que haya transparencia acerca de quiénes son los responsables de aconsejar a las víctimas, recibir y trabajar las quejas, de cómo llevar a cabo las investigaciones, imponer castigos y proteger a la persona afectada y a otras presuntas víctimas mientras la investigación esté en curso.

En 2015 se analizaron 24 de estos protocolos, con particular énfasis en 12, para identificar ejemplos, modelos y lagunas de protección persistentes (Kocher \& Porsche, 2015). ${ }^{16}$ Los protocolos varían de dos a 17 páginas y se diferencian por los puntos regulados; lo que todos tienen en común es que en la definición del HAS y en el argumento de tener que proteger a las integrantes de la institución se basan en el artículo 3, párrafo 4 de la AGG (2006, p. 29). En varios protocolos se aclara específicamente que la atención debe abarcar a todas y todos los integrantes de la universidad, es decir, también a las y los estudiantes y que se debe de dar a conocer al público. En un protocolo se especifica que la violencia sexualizada también puede ser efectuada por miembros del cuerpo estudiantil y que, por lo tanto, incluye las relaciones entre estudiantes, pero en ninguno se detalla que en su función de protección abarquen a las personas LGBIT,* porque ellas también son dañadas por este tipo de violencia; sin embargo, en varias universidades en la práctica se les toma en cuenta.

En cuanto a los procedimientos, los protocolos más amplios están distinguiendo entre un procedimiento de queja informal y uno formal (AGG, 2006, p. 33). La atención a la informal comprende dar información sobre los diferentes canales de queja y asesoría psicológica y jurídica; la vía formal se inicia con una queja escrita y entregada a la persona u oficina responsable. Dos principios muy importantes del procedimiento (informal y formal) que se citan en muchos de los protocolos son la confidencialidad y la prohibición de sanciones disciplinarias, es decir, la garantía para las personas afectadas de que el hecho de entregar una queja no les va a provocar problemas o sanciones en la universidad.

En cuanto a las medidas de castigo, se hace una diferencia entre si el agresor es empleado, estudiante o si es de fuera de la institución y van desde los apercibimientos hasta la exmatriculación, despido o la prohibición de volver a entrar a la universidad. En todos los casos, al final del proceso existe la posibilidad de que el rectorado presente una denuncia ante la policía o la fiscalía (Kocher \& Porsche, 2015, p. 43).

En cuanto al diseño de los protocolos, la coordinadora -basándose en dicho análisis- emite las siguientes recomendaciones (Bukof, 2018b):

- Que los protocolos abarquen a todos los grupos que se encuentran en la universidad, es decir, también a los estudiantes, quienes

15 En Alemania hay 82 millones 790 mil habitantes, de los cuales 2 millones 87 mil son estudiantes (Statista, 2018). De las 415 IES, 106 son universidades, seis son escuelas superiores de Pedagogía, 16 de Teología, 51 de Arte y 207 de otras especialidades; por ejemplo, técnicas o de salud y 29 de carreras administrativas (Kocher \& Porsche, 2015, p. 27).

${ }^{16}$ Además, se entrevistó a las personas responsables del tema en las IES, sobre todo a las delegadas de mujeres, para comprender más a fondo las problemáticas que ahí se registran en torno al tema. No es una investigación representativa, sino más bien explorativa que puede servir para mejorar los protocolos existentes y desarrollar nuevos. 
no tienen contrato de trabajo (sólo contrato de honorarios y por ello no son considerados "empleados"), las personas de diversidad sexual/LGBIT* y los varones.

- Con lo anterior, cerrar los huecos de protección persistentes.

- Que las universidades implementen un procedimiento de quejas que sea transparente y fácil; y que se instalen oficinas responsables de recibir y atender las quejas.

- Que estas oficinas sean estructuras profesionales de asesoría psicológica y jurídica, con personal capacitado.

- Que en los protocolos se exprese la garantía de absoluta confidencialidad (y que esto se cumpla en la realidad), para proteger y ganar la confianza de las personas afectadas, para así aumentar el número de quejas y bajar el nivel de impunidad.

- Que haya cursos de capacitación obligatorios sobre el tema dirigidos al personal de dichas oficinas y a los directivos, como por ejemplo, directoras de institutos y facultades. Esto es, que el tema se integre a los mecanismos y procedimientos del desarrollo de la organización.

- Que se ofrezcan cursos de capacitación para empleadas y estudiantes interesadas en el tema.

- Que se instalen diversos mecanismos de sensibilización y prevención.

- Que se establezca un grupo de trabajo permanente acerca del tema con la participación de representantes de todos los sectores universitarios para monitorear y evaluar los protocolos y otros mecanismos.

- Que las universidades faciliten los recursos adecuados y necesarios para hacer todo lo arriba mencionado.

\section{Otros instrumentos de sensibilización e intervención}

Para fomentar una cultura institucional libre de discriminación se están usando diversos instrumentos de sensibilización. Como ya se dijo antes, los mismos protocolos y también las investigaciones pueden contribuir a este fin, dando a conocer las medidas de protección, a los responsables y los procedimientos de queja, asesoría, investigación y sanción, además de recabar datos sobre la problemática en la misma IES.

\section{Trabajo de investigación}

Se están levantando encuestas en las universidades para obtener información que sirva para comprender mejor el problema, para diseñar medidas idóneas y -al momento de aplicar la encuesta y de publicar los resultados- para sensibilizar (Kocher \& Porsche, 2015, p. 40); un ejemplo es el proyecto de investigación transnacional "Discriminación y violencia sexualizada en las escuelas superiores en el contexto internacional", en el que coopera la FU Berlín con universidades de Costa Rica, Colombia, Perú, Ecuador, India, Japón, Corea y la UNAM. Asimismo, hay otras encuestas en las IES sobre las experiencias de las y los estudiantes con el HAS y su percepción del tema. ${ }^{17}$ Un resultado intermedio de la FU Berlín es que las reacciones de los profesores al entrar a los cursos y aplicar el cuestonario son muy diversas, y también la percepción del tema por parte de los estudiantes resulta muy variada: desde respuestas sexistas hasta solidarias (Lawrenz, 2018, p. 42).

Otro instrumento consiste en la difusión del problema a través de tarjetas y volantes, páginas web y eventos. Estos materiales y medios de informacion

\footnotetext{
${ }^{17}$ https://www.lai.fu-berlin.de/institut/gremien/frauenbeauftragte/Projekt-_Sexualisierte-Diskriminierung-und-Gewalt-an-Hochschulen_imtransnationalen-Kontext/index.html

https://www.lai.fu-berlin.de/forschung/Konferenzen/Veranstaltungen_2018
} 
tienen dos efectos: primero, orienta a las posibles víctimas de sus derechos y de dónde encontrar asesoría, y segundo, es una señal hacia los potenciales agresores de que en la universidad no se acepta la DVS y que será sancionada. Por ejemplo, en la FU Berlín, en octubre de 2017, se organizó una semana temática contra la DVS titulada "No significa No", con mesas redondas, películas y discusiones, se distribuyeron volantes y contenido en la página web de la delegada de la mujer. ${ }^{18}$ En este contexto también se publicó un video hecho por estudiantes sobre violencia de género (Wolff, 2017). ${ }^{19}$

\section{Material de información}

Otro ejemplo son los carteles diseñados por la coordinadora, los cuales todas las delegadas del país pueden usar para su trabajo, añadiendo los datos de contacto específicos de su institución. ${ }^{20}$ Además, en su página web la coordinadora publica material de orientación sobre el tema, tanto para personas afectadas como para quienes brindan apoyo. ${ }^{21}$

Otras medidas de sensibilización y prevención pueden ser:

- Cursos de autodefensa y de empoderamiento para grupos vulnerables.

- Cambios infraestructurales en los edificios y en el campo universitario.

- Eventos culturales al respecto.

- Anuncios verbales en las juntas de las facultades e institutos.

- Acciones autónomas de estudiantes y activistas feministas.

\section{Estrategias y acciones "de base"}

Ante las problemáticas de los mitos de "violación"22 (Sanyal, 2016; Wiezorek, 2014) y del estigma que todavía se relaciona con la DVS, y considerando la falta de confianza en los procedimientos de queja y la alta impunidad, (no solamente) las estudiantes alemanas han ido diseñando estrategias y acciones independientes, informales y "de base". Entre ellas, se están estableciendo grupos de intercambio de experiencias, weblogs o grupos en Facebook para compartir y publicar anónimamente lo vivido, se difunden volantes y carteles para advertir a las demás estudiantes de presuntos violadores, se producen videos, organizan plantones y manifestaciones, eventos culturales o discusiones políticas, refiriéndose a casos concretos en las IES. Sobre estas estrategias en el ámbito universitario todavía no hay investigación, solamente uno que otro ejemplo. En el documental "La mano en el trasero" (Greaf, 2018) se refieren dos ejemplos: el primero es el de varias estudiantes que sufrieron DVS de un mismo docente y que se reunieron a intercambiar sus experiencias; con el tiempo, se animaron a reportar los casos, se apoyaron mutuamente en el proceso y lograron que se despidiera al docente; sin embargo, por fallas formales de parte de la universidad, el inculpado termina destituido, pero con un pago de indemnización y un certificado de trabajo. Segundo, en un blog las víctimas de DVS pueden reportar de manera anónima, lo que les da oportunidad de escribir sobre sus experiencias y compartirlas con otras personas que las entiendan y respeten. En el mismo proceso de reflexionar escribiendo, de hacer público lo vivido, posiblemente las autoras encuentren algo de alivio. Al

${ }^{18}$ Véase: https://www.fu-berlin.de/sites/nein-heisst-nein/faq/index.html

19 Con la participación de estudiantes de habla española, véase: https://www.youtube.com/watch?v=0QGGDJ3I9KM

20 Véase: https://bukof.de/wp-content/uploads/bukof-Postkarte-SDG.pdf

21 Véase: https://bukof.de/online-handreichung-sdg/\#1492508190062-38ff2c70-ee02

22 El concepto "mitos de violación" puede ayudar a explicar cómo este delito en la opinión pública se está minimizando de tal manera que más bien se considera una bagatela, disculpando a los agresores y señalando a las víctimas. Estos mitos, además, tienen el efecto que las sobrevivientes tienden a culparse a sí mismas y por esta razón no denuncian o porque les da vergüenza. Los mitos son, por ejemplo, que solamente las mujeres jóvenes y/o las que se apegan al ideal de belleza dominante son violadas, que se les viola porque su forma de vestir "invita" o "provoca" a los violadores, que una violación solamente es una forma de sexualidad un poco más fuerte o violenta, que a los hombres no se les puede violar porque ellos siempre quieren tener sexo, que no puede ser una violación si las dos personas ya tuvieron sexo en otra ocasión, que a las sexoservidoras no se les puede violentar, que la mayoría de las violaciones suceden en la noche en un parque y que los violadores padecen de perturbaciones psicológicas o de sexualidad. Se entiende que todos estos mitos no tienen que ver con la realidad (Wiezorek, 2014, p. 112 y siguientes; Sanyal, 2016, p. 40 y siguientes). 
mismo tiempo, están creando una atención pública que ayuda a superar el tabú y enviar el mensaje de que la DVS ya no se va a tolerar. El blog para las víctimas es un espacio donde no se duda de ellas ni se les culpa por lo que les pasó (Graef, 2018).

Algunas estrategias activistas (como blogs y carteles identificando a los presuntos agresores) pueden ser concebidas como una violación a los derechos personales de los señalados; al mismo tiempo, pueden ser vistas como un SNAP, como lo llama la filósofa inglesa Sara Ahmed (2017), es decir, una acción "explosiva" por la que optan las personas cuando ya no aguantan la discriminación y violencia vivida, el desinterés de la sociedad y la impunidad de los agresores. Estoy convencida de que cuando ya funcionen los mecanismos institucionales y las vías formales de protección, intervención y sanción, las conductas de este tipo se resolverán.

\section{Sugerencias subjetivas}

Sumándome a las sugerencias de la coordinadora acerca de los protocolos y partiendo de lo escrito arriba y de mis observaciones en la práctica, personalmente quiero enfatizar los siguientes puntos y añadir otras recomendaciones.

Pienso que deberíamos, en especial, empoderar a las personas que corren el riesgo de ser afectadas, por ejemplo, a través de talleres de autodefensa o grupos de conversación y autoayuda, animarlas a que en situaciones críticas se atrevan a decir "no", a expresar y cuidar sus límites y a defenderse. Segundo, deberíamos impulsarlas y apoyarlas a que busquen ayuda psicológica y jurídica dentro o fuera de las IES.

En los procedimientos, hay que garantizar confidencialidad absoluta. Si la víctima decide poner una queja formal en el centro educativo o ante la policía, habrá que darle seguimiento y consejería capacitada y empática. Además, hace falta establecer reglamentos para proteger a quienes ponen una queja para que se les proteja de agresiones por el hecho de haberla presentado. En los casos de denuncias severas dentro de la IES (como por persecución y violación), habrá que procurar que los acusados o acusadas ya no tengan contacto con gente vulnerable, como estudiantes o con subalternos, hasta que concluya la investigación. Lo anterior, con el fin de demostrar a la comunidad universitaria en general que la institución actúa según su responsabilidad de proteger a todos y para ganar confianza en cuanto a que las denuncias son tratadas con cuidado y seriedad.

A los estudiantes que salen a hacer prácticas, investigaciones de campo, excursiones 0 que participan en programas de intercambio a nivel nacional e internacional, habrá que darles información y preparación adecuada. $Y$ en los protocolos habrá que incluir reglamentos que se deben acordar con las organizaciones e instituciones de cooperación, nacionales e internacionales.

Es razonable respetar las estrategias de las activistas y estudiantes que no están previstas dentro de las vías institucionales, porque pueden ser una manera de asimilar y trabajar lo sufrido, además de que también son un instrumento de romper con el tabú y de sensibilizar acerca del problema; es decir, es apropiado practicar una solidaridad feminista que nos permita apoyarnos mutuamente, en nuestro empeño por una cultura pacífica, libre de discriminación y violencia.

Hay que considerar la intersección de diferentes tipos de discriminación, a fin de diseñar mecanismos y servicios adecuados para necesidades y situaciones diversas y específicas. Al mismo tiempo, habrá que tomar en cuenta que el tema de la DVS puede ser instrumentalizado, por ejemplo, por grupos racistas y nacionalistas. Entonces habrá que impedir que la discriminación de un grupo de personas (por ejemplo, los refugiados) se justifique con la presunta protección de otro (las mujeres, por decir alguno). ${ }^{23}$

\footnotetext{
${ }^{23}$ Véase nota a pie 3.
} 
Además, no debemos olvidar que puede haber estudiantes que ya vivieron violencia sexualizada en otros contextos y que al nuevamente encontrarse con ella en la universidad pueden ser revictimizadas y retraumatizadas, ${ }^{24}$ por lo tanto, urge establecer servicios y mecanismos especiales para responder a esta problemática. En especial, en las disciplinas de Ciencias Sociales, Educación, Humanidades y Derecho, pero al fin y al cabo en los currículos de todas habrá que implementar cursos acerca del tema. Esto, con el fin de sensibilizar y capacitar a los estudiantes, que luego serán personas con responsabilidad en sus puestos de trabajo fuera de la universidad, en los diferentes ámbitos de la sociedad. ${ }^{25}$

Para finalizar, habrá que promover la investigación sobre el asunto, por ejemplo, las encuestas en las IES para recabar más datos y diseñar medidas adecuadas o indagaciones sobre el fenómeno de la revictimización en el estudiantado, sobre la intersección de la DVS con otras formas de discriminación y violencia (como el clasismo, el racismo, etcétera) o sobre las acciones alternativas, no institucionales, feministas.

\section{Conclusiones}

La discriminación y violencia sexualizada provoca interrupciones en la formación profesional de las integrantes de las IES, afectaciones y rupturas en sus vidas en general. Ya es hora de erradicarla y ya estamos -a nivel mundial y a pesar de la reacción autoritaria y derechista en algunos de nuestros países- en vías hacia una vida académica (y cotidiana) libre de estas conductas. Como se mostró en los capítulos anteriores, en Alemania vamos avanzando, poco a poco: se están tomando y diseñando acciones de base para sensibilizar acerca del tema y para apoyarse mutuamente. Las delegadas de mujeres, ${ }^{*}$ junto con algunas estudiantes, empleadas académicas y administrativas están fomentando las campañas de información, los proyectos de investigación y trabajando por implementar los protocolos como base jurídica segura para todas las integrantes de la universidad. La Conferencia de los rectores de las IES alemanas en 2018 se pronunció a favor de instalar los protocolos y también en los medios masivos de comunicación (gracias a los movimientos \#metoo, \#ni una menos, \#aufschrei y \#ausnahmslos) se empieza a abordar de otra manera el tema y a considerar la convergencia de diferentes tipos de discriminación.

El caso del ex presidente de la Universidad de Música y Teatro de Munich, Siegfried Mauser, quien fue condenado a dos años y nueve meses de cárcel por acoso sexualizado a tres personas puede motivar para tal vez ganar más confianza en el instrumento de la denuncia penal26 (Graef, 2018).

Sin embargo, todavía nos quedan bastantes problemas que resolver y cimas (además de alguna que otra montaña) que sobrepasar. Entra ellas está el doble tabú en torno de la probemática y la alta impunidad (no solamente) en las IES. Ahí están las estructuras y culturas (heterosexistas) que llevan a que: 1 ) los agresores no conciban como agresión a sus actos violentos, 2) las personas afectadas no sepan o no crean en su derecho a decir "no", y 3) en el caso de que denuncien la agresión, ellas mismas son calificadas como agresoras o perturbadoras, lo cual 4) conduce a represiones y nuevas agresiones en su contra. Están los desafíos de la falta de información y sensibilización en las IES, la falta de credibilidad (y decisión) de las instancias universitarias que reciben las quejas $y$

${ }^{24}$ Que yo sepa, hasta ahora no hay investigaciones acerca de la problemática en las IES alemanas, pero existen estudios del contexto de la pedagogía y el trabajo social que parcialmente pueden indicar medidas necesarias también para las IES (véase Helfferich et al., 2019).

${ }^{25}$ Retkowski et al (2019) presentan un currículo básico sobre "Violencia sexualizada en las instituciones" para las disciplinas de Pedagogía, Trabajo social y la formación de maestros de primaria, secundaria y preparatoria en Alemania.

${ }^{26}$ La sentencia todavía no está firme, porque tanto el acusado como una querellante (una de las tres afectadas que pusieron una denuncia) interpusieron recurso de casación: el acusado repite su inocencia mientras la querellante quiere probar que también hubo una violación y no "solamente" los acosos sexualizados (Wiegand \& Wimmer, 2018). 
realizan las investigaciones, la falta de capacitación del personal responsable, de oficinas o de quienes dan asesoría jurídica y psicológica, recursos insuficientes en las oficinas, datos sobre el problema y de fondos para la investigación, la falta de la implementación del tema en los currículos universitarios y la desigualdad de poder que dificulta que las estudiantes se puedan defender de las agresiones de sus docentes y compañeros, entre muchas otras limitantes.

Pero ahí vamos, porque, entre otras cosas, nos motiva el ejemplo de nuestras antepasadas que ya lograron el derecho de las mujeres* a ingresar a las universidades, el derecho a votar y a participar en la vida política y pública. Y porque una vida libre de discriminación y violencia es una buena vida y vale la pena ( $\mathrm{y}$ a veces hasta es divertido) luchar por ella.

\section{Referencias}

Ahmed, S. (2017). Living a Feminist Life. Durham: Duke University Press.

BMFSFJ (Bundesministerium für Familie, Senioren, Frauen und Jugend) (Ed.). (2008). Lebenssituation, Sicherheit und Gesundheit von Frauen in Deutschland. Eine repräsentative Untersuchung zu Gewalt gegen Frauen in Deutschland. Zusammenfassung zentraler Studienergebnisse. Berlín: autor.

Butler, J. (2004). Undoing Gender. New York: Routledge.

Eberlei, W., Neuhoff, K. \& Eberlei, R. K. (2018). Soziale Arbeit mit Betroffenen von Sexualisierter gewalt. En Dies: Menschenrechte-Kompass für die soziale Arbeit (pp. 86-97). Stuttgart: Kohlhammer Verlag.

Feltes, T., List, K., Schneider, R. \& Höfker, S. (2012). Gender-based Violence, Stalking and Fear of Crime. Länderbericht Deutschland: Bochum.
Helfferich, C., Kavemann, B., Kindler, H., Nagel, B. \& Schürmann-Ebenfeld, S. (2019). ReViktimisierung nach sexuellem Missbrauch in einer Hochrisikogruppe. Ergebnisse einer Mixedmethod-Studie bei Mädchen und jungen Frauen in stationären Einrichtungen der Jugendhilfe. En M. Wazlawik, H.-j. Voß, A. Retkowski, A. Henningsen \& A. Dekker (Ed.), Sexuelle Gewalt in pädagogischen Kontexten. Aktuelle Forschungen und Reflexionen (pp. 55-70). Wiesbaden: Springer VS.

Kocher, E. \& Porsche, S.(2015). Sexuelle Belästigung im Hochschulkontext-Schutzlücken und Empfehlungen. Berlín: Antidiskriminierungsstelle des Bundes.

Retkowski, A, Dekker, A., Henningsen, A., Voß, H.J. \& Wazlawik, M. (2019). Basis-Curriculum zur Verankerung des Themas "Sexuelle Gewalt" in Institutionen in universitärer und hochschulischer Lehre. En M. Wazlawik, H.-J. VoB, A. Retkowski, A. Henningsen \& A. Dekker (Ed.), Sexuelle Gewalt in pädagogischen Kontexten. Aktuelle Forschungen und Reflexionen (pp. 261-290). Wiesbaden: Springer VS.

Sanyal, M. M. (2016). Vergewaltigung. Aspekte eines Verbrechens. Hamburg: Edition Nautilus.

Seifarth, S. y Ludwig, H. (2016). Dunkelfeld und Anzeigeverhalten bei delikten gegen die sexuelle Selbstbestimmung. Ergebnisse eienr Untersuchung zur Erforschung von Anzeigemotivation und Anzeigeverhalten bei sexueller Nötigung und Vergewaltigung. Monatsschrift für Kriminologie und Strafrechtsreform 99, 237-242.

Stadler, L., Bieneck, S. \& Pfeiffer, C. (2012). Repräsentativbefragung Sexueller Missbrauch 2011. Forschungsbericht Nr. 118. Hannover: Kriminologisches Forschungsinstitut Niedersachsen. 
Wiezorek, A. (2014). Weil ein \#Aufschrei nicht reicht. Für einen Feminismus von heute. Frankfurt/ Main: Fischer Verlag.

\section{Webgrafía}

AGG-Allgemeines Gleichbehandlungsgesetz. (2006). Recuperado de https://www.gesetze-iminternet.de/agg/BJNR189710006.html

Bff-Frauen gegen Gewalt e.V. (s.f). Zahlen und Fakten zum Plakat "Vergewaltigung verurteilen". Recuperado de https://www.frauen-gegengewalt.de/de/zahlen-und-fakten-zum-plakatvergewaltigung-verurteilen.html

Bukof. (2018a). Grundsatzpapier zu sexualisierter Diskriminierung und Gewalt an Hochschulen. Berlin: bukof. Recuperado de https://bukof.de/wp-content/ uploads/2018-Grundsatzpapier-SDG.pdf

Bukof. (2018b). Pressemitteilung: Für einen verantwortungsbewussten Umgang mit sexualisierter Diskriminierung und Gewalt an Hochschulen. Berlin: bukof. Recuperado de https:// bukof.de/wp-content/uploads/18-05-24-bukofPressemitteilung-SDG-an-Hochschulen.pdf

Bukof. (2018c). Online Handreichung Sexualisierte Diskriminierung und Gewalt an Hochschulen. Richtlinien an Hochschulen-Beispiele. Recuperado de https://bukof.de/online-handreichungsdg/\#1492508909531-846a2027-1aee

Conferencia/Hochschulrektorenkonferenz. (2018). Empfehlung der 24. Mitgliederversammlung der HRK am 24.4.2018 in Mannheim. Gegen sexualisierte Diskriminierung und Belästigung im Hochschulkontext. Berlín: HRK. Recuperado de https://www.hrk.de/fileadmin/redaktion/ hrk/02-Dokumente/02-01-Beschluesse/HRK_MV_ Empfehlung_sexBelaestigung_24042018.pdf
FRA (EU-Grundrechte-Agentur /European Union Agency for Fundamental Rights). (2013). Erhebung unter Lesben, Schwulen, Bisexuellen und Transgender-Personen in der Europäischen Union. Recuperado de: https://fra.europa.eu/ de/publication/2014/lgbt-erhebung-der-euerhebung-unter-lesben-schwulen-bisexuellenund-transgender

Klein, U. \& Rebitzer, F. (2012). Vielfalt und Diskriminierungserfahrungen: Ergebnisse einer Befragung der Studierenden an der CAU. Kiel: Universität Kiel. Recuperado de https://www. gendiv.uni-kiel.de/de/forschung/downloads/2012cau-studierendenbefragung.pdf

Lawrenz, N. (2018). Diskurse und Praktiken sexualisierter Diskriminierung und Gewalt an Hochschulen. Ein internationales Forschungsprojekt der Freien Universität Berlin mit Universitäten in Lateinamerika und Asien. En Korueber, Mechthild/Zentrale frauenbeauftragte der FU Berlin: Wissenschaftlerlnnen-Rundbrief 1/2018. Berlín: FU Berlín. S. 40-43. Recuperado de https://www.fu-berlin.de/sites/frauenbeauftragte/ media/WRB_012018_Web.pdf

Oerter, D., Lorenz, S. \& Kleine, I. (2012). Auswertung der Social media Kampagne \#ichhabenichtangezeigt. Recuperado de https://ichhabnichtangezeigt.files.wordpress. com/2012/07/auswertung_ausf-web.pdf

Statista-Das statistikportal. (2018). Studierendenzahlen in Deutschland 2017/18. Recuperado de https://de.statista.com/statistik/ daten/studie/221/umfrage/anzahl-der-studentenan-deutschen-hochschulen/

Wiegand, R. \& Wimmer, S. (2018). Ex-Präsident der Münchner Hochschule für Musik muss in 
Haft. Süddeutsche Zeitung vom. Recuperado de https://www.sueddeutsche.de/muenchen/prozessmauser-haft-urteil-1.3983187

\section{Videos}

Graef, N. (2018). Die Hand am Po. Un documental de los canales WDR y ARD. Colonia. Recuperado de https://www.daserste.de/information/reportagedokumentation/dokus/sendung/die-hand-ampo-100.html
Wolff, M. (2017). Gender based violence and discrimination at Universities. FU Berlín, Berlín. Recuperado de https://www.youtube.com/ watch?v=0QGGDJ3I9

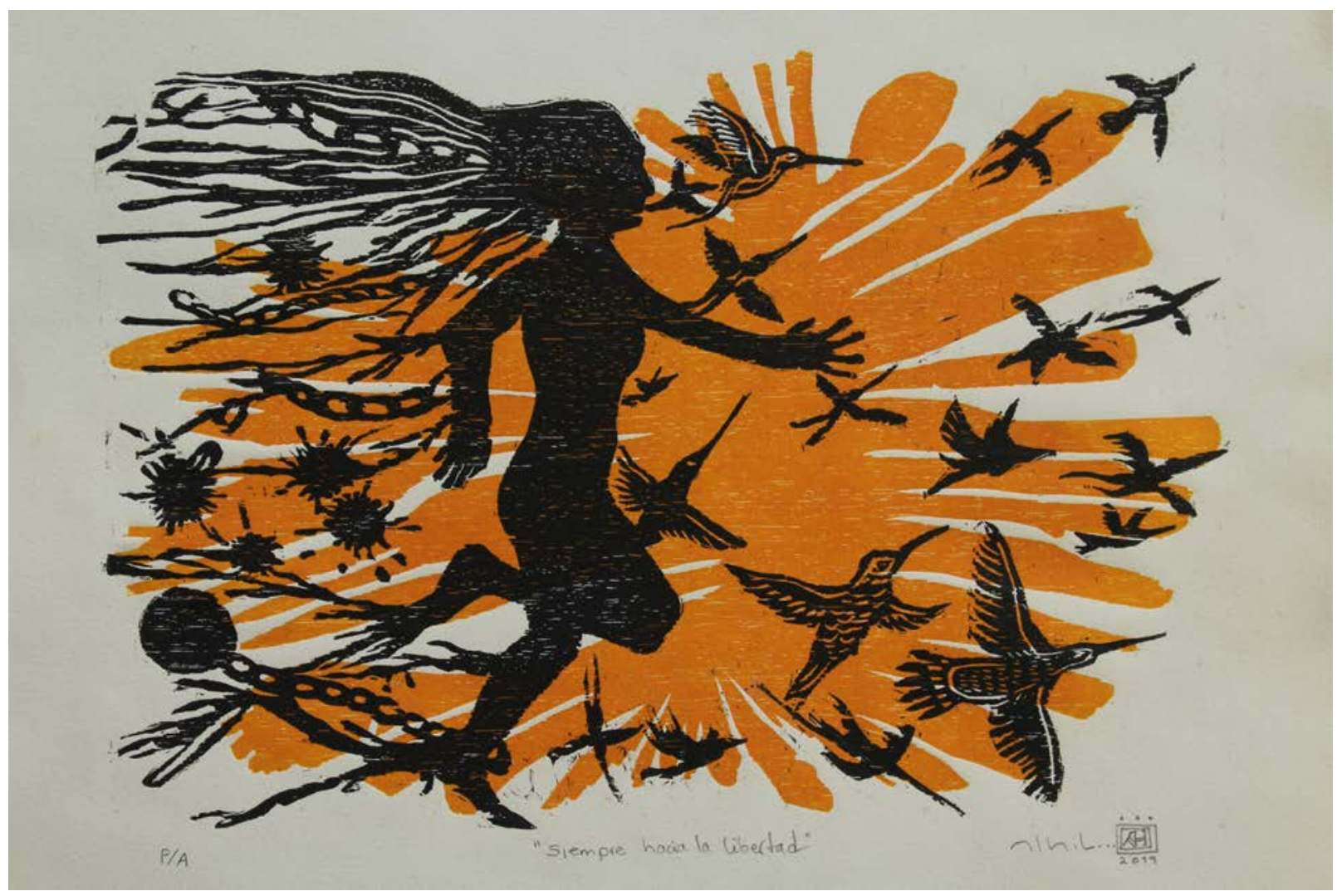

"Siempre hacia la libertad" Xilografía a dos tintas sobre papel de algodón $30 \times 40 \mathrm{~cm}$ 2019 


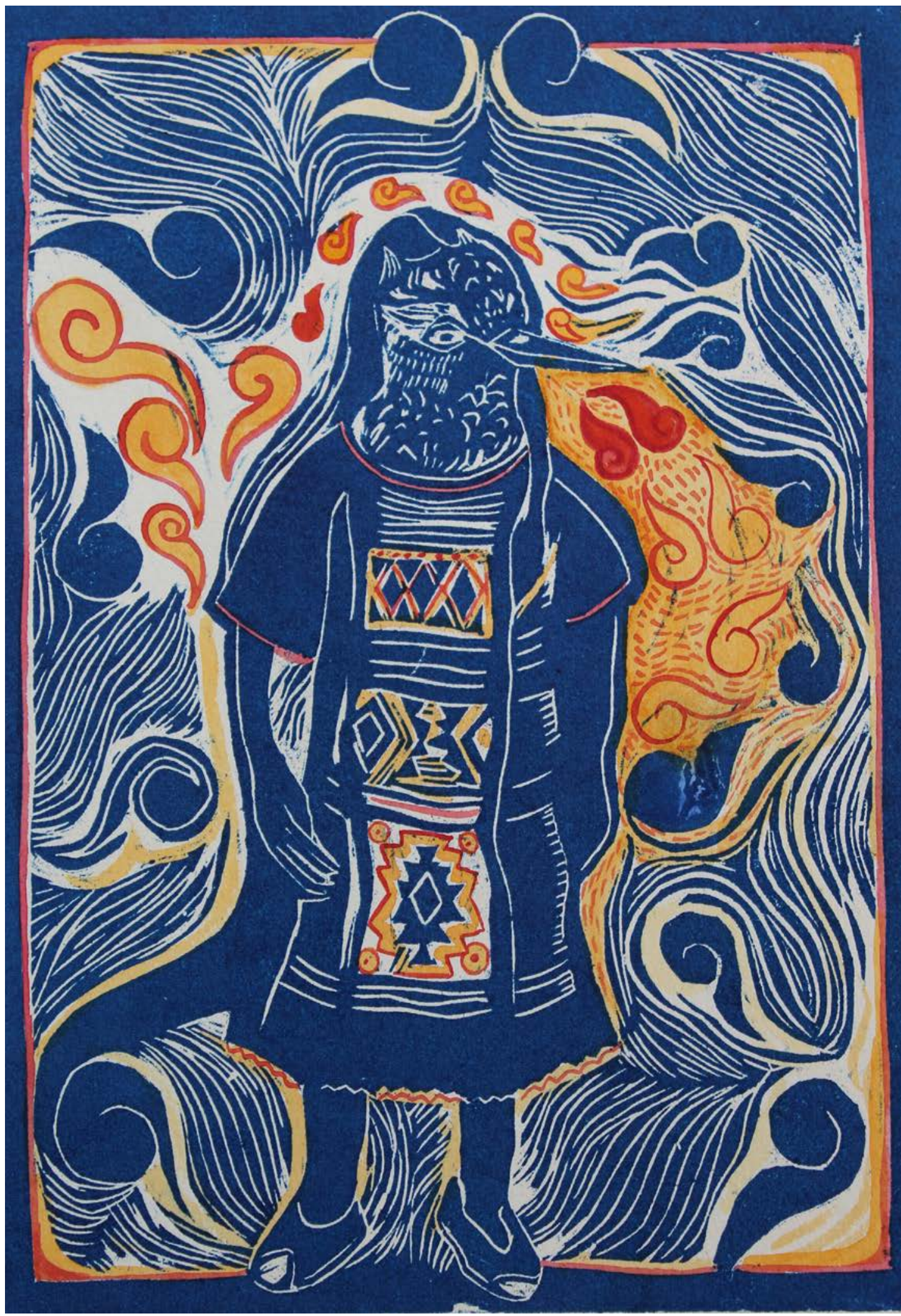

"Cantos de libertad"

Linóleo

$30 \times 20 \mathrm{~cm}$

2018 